\title{
Use of absolute function and its associates in formation and 'redevelopment' of mathematical models in some plant-related quantitative physiology: salinity effects on leaf development of Schefflera arboricola and harvest index in rice
}

\begin{abstract}
The roles of quantitative physiology are becoming more apparent and crucial in the era of ICT recently. As based on the rate-related variables, most of the mathematical models are in the form of 'non-linear' function in describing the responses or the observed within-plant processes outcomes versus time. Even though if some responses change in a drastic manner at certain response point within a biological unit or space of a plant system, the response curve 'should' be dependent on a continuous independent variable range in a specified period of determination where biologically 'should not' functioned by independent variable range having 'IF' statement(s). Subjected to nutrient concentration of high salinity $(6.0 \mathrm{mS} \mathrm{cm}-1)$, the leaf turgidity (measured as leaf surface area) of S. arboricola which initially was described by one form of the logistic growth functions $[(y=1 /(a+b e-c x)]$ abruptly reduced as explained by a model having terms of Absolute function (ABS) containing tan-1(x) and its parameter of leaf life expectancy as affected by high salinity growing medium at a certain point of days after planting. This yielded an overall function of $\left.\mathrm{y}=1 /(\mathrm{a}+\mathrm{be}-\mathrm{cx})-\mathrm{A}\left[\tan ^{-1} 1(\mathrm{x}-\mathrm{B}) / \mathrm{D}\right\}+\mathrm{ABS}\left(\tan ^{-1} 1\{(\mathrm{x}-\mathrm{B}) / \mathrm{D}\}\right)\right] \mathrm{E}$, where $\mathrm{a}, \mathrm{b}, \mathrm{c}, \mathrm{A}, \mathrm{B}, \mathrm{D}$, and $\mathrm{E}$ are constants that most of them can be 'biologically' interpreted. The constant $\mathrm{B}$ is the point similar to 'IF statement' as normally used in other mathematical functions. Plants subjected to lower salinity status $(<3.0 \mathrm{mS} \mathrm{cm}-1)$ were only having function of $y=1 /(a+b e-c x)$. In the harvest index or HI (economic yield/above ground biomass) study of 20 rice varieties grown over two planting seasons, the long flattened tails at both sides of a peak in the middle of function of $y=R+B(T+A B S(B-x)) e-k(T+A B S(B-x))$ had indicated that those varieties maturing at 123 to 133 days after transplanting were having high $\mathrm{HI}$ values. In our observation, Absolute (ABS) function coupled with some terms could be used in the formation of some mathematical functions having biological meaningful constants in explaining some quantitative physiological and biological responses vs. independent variables studied.
\end{abstract}

Keyword: Absolute function; Built-in IF-statement; Mathematical models; Quantitative physiology 